\title{
The effect of daily walking exercise on sleep quality in healthy young adults
}

\author{
Feifei Wang ${ }^{1}\left[\right.$ D $\cdot$ Szilvia Boros ${ }^{1} \mathbb{D}$
}

Received: 8 April 2020 / Accepted: 22 September 2020 / Published online: 24 December 2020

(c) The Author(s) 2020

\begin{abstract}
Purpose Walking has beneficial effects on sleep quality in elderly population and patients in clinical settings. However, less is known whether walking improves sleep quality among healthy young adults. This study examined the effectiveness of a 12 -week walking intervention on sleep quality among sedentary young adults.

Methods Fifty-four healthy adults aged 19 to 36-years old participated a pedometer based aerobic walking intervention, a cross-over randomized control trial. Participants were assigned into two groups (group A and group B) randomly. The 12-week intervention was divided into three sessions equally. Sleep quality was assessed by Pittsburgh Sleep Quality Index (PSQI) before and after session 1 and session 3. Omron HJ-112 pedometer and daily walking diary facilitated the intervention process. Within group and between group comparisons were made for statistical analysis.

Results Within group comparison by repeated measures showed that sleep duration $(p<0.01, F$-test 22.79$)$, sleep medication $(p<0.05, F$-test 5.22), subjective sleep ( $p<0.05, F$-test 5.51) and global sleep quality $(p<0.01, F$-test 12.19$)$ were significantly improved. The comparison between intervention group and control group showed that sleep disturbance was significantly improved $(p<0.05)$.

Conclusion Daily walking exercise has a significant effect on facilitating sleep quality and sleep components among young adults. Further studies are suggested to examine the impact of walking intensity on sleep quality.
\end{abstract}

Keywords Physiotherapy $\cdot$ PSQI $\cdot$ Sleep intervention $\cdot$ Physical activity $\cdot$ Aerobic

\section{Introduction}

Sleep disorders are highly prevalent in recent years. Evidence from epidemiologic studies has uncovered the severity of sleep disturbance and high prevalence of undiagnosed sleep disorders [1, 2]. A frequent compliant of sleep disorder is insomnia; the National Institutes of Health (NIH) estimated that roughly $30 \%$ of the general population suffer from sleep disruption [3]. It was reported that a large scale of adults has difficulty in initiating and maintaining sleep [4]. Sleep disorder becomes an increasing concern in modern society, it is of importance to identify intervention strategies in order to decrease and narrow down sleep disorders.

Feifei Wang

feifei.wang@ppk.elte.hu

1 Institute of Health Promotion and Sport Sciences, Faculty of Education and Psychology, ELTE Eötvös Loránd University, Budapest, Hungary
Sleep is crucial for adults to manage work-life balance and life satisfaction; sleep is regarded as an important marker of life quality [5]. Due to the increase of sleep disorders, academic research investigating sleep interventions demonstrated that sleep complaints benefit from exercise. Both previous and recent study revealed that sedentary people were more closely associated with poor sleep compared with physically active people $[6,7]$. Moreover, physical exercise was emphasized in sleep management programs [8]. Nevertheless, both the intensity and the timing of exercise as well as exercise duration should be assessed, also, the frequently used sleep measurements include polysomnography, actigraphy, questionnaires, etc. [9-11]. At present, the relationship between physical activity intensity and sleep improvement is still argumentative. Early study declared that moderate exercise had positive effects on sleep efficacy and sleep behavior $[12,13]$, as well as in pathological conditions such as cancer and metabolic dysfunctions [13, 14]; in addition, late night vigorous exercise increased negative impacts on sleep $[15,16]$. To our best knowledge, there was only one 
article supports that vigorous exercisers have more favorable effects on objective sleep pattern in young adults [17]. Considering the feasibility and applicability for public health implementation, sleep complaints need to be approached by activities that have longer-term, sustained benefits and with preference to limit pharmacological intervention as far as practicable [18].

With the evidence suggesting that moderate physical intensity may be more beneficial in young adults, a variety of experimental studies have examined structured physical activity to promote sleep quality. For instance, yoga showed significant benefits to improve sleep quality, and can be incorporated as an activity program in sleep intervention [19]. Additionally, Tai chi appears to be effective as a nonpharmacological approach to enhance sleep [20]. The results of aquatic bio-dance also contributed to improvements in sleep quality [21]. Besides, a group of young adults (age between 18 and 30 years old) who participated in a Pilates exercise program experienced improvement in sleep quality and life quality [22]. However, regarding to population level health promotion, those interventions mentioned above may not feasible and realistic for everyone.

Walking is recognized as sustainable exercise that does not need professional instructions to perform, which allows the possibility in public health promotion. Walking in nature has been shown to be effective to enhance mental well-being [23]. A before and after comparison regarding to forest walking supported that forest walking improved nocturnal sleep conditions among a community-based sample of people with sleep complaints [24]. Meta-analyses have exploited the benefits of moderate-intensity walking for clinical patients $[25,26]$, and moderate-intensity walking exercise showed efficiency in improving sleep [27]. Brisk walking lasting for more than at least $20 \mathrm{~min}$ is associated with less subsequent sleep difficulty [28] and it is recommended to add walking exercise program to the daily program for elderly population [29]. However, a randomized controlled trial worked on walking and light exposure, stated that walking showed no significant effect on improving sleep disorder [30]. What are the reasons for the adverse results? Covariables (e.g. health status, age) could affect sleep outcome, which may confound the intervention effect. Even though the number of walking-based exercise interventions is increasing, but most of studies were done among patients with symptoms or among elderly people, limited evidence was found among healthy young adults [31, 32]. Plus, increasing physical level of adults has becomes a hot topic in improving public health [33]. Based on these concern, supplementary evidence of this topic is needed.

Given the existed evidence that the effect of walking exercise on sleep among young adults is under exploration, plus, it lacks of evidence whether walking benefits sleep quality among young adults, there is a demand to explore the notability of walking intervention. In particular, this study aimed to investigate the effectiveness of aerobic walking exercise on sleep quality and sleep related components among healthy young adults.

\section{Methods}

\section{Study design}

A crossover intervention study was conducted with a total of 54 participants recruited between January 2019 and February 2019 through social media advertisements and from call-for-volunteer posters in faculties in Eötvös Loránd University (ELTE), Hungary. The preparation of the study includes ethical approval, pedometer purchase, and intervention procedure assessment, participant coordination. The research protocol was approved by the ethical committee in Faculty of Education \& Psychology in ELTE. Selected participants have signed the written consent form and all of the participants were orientated before starting intervention. The intervention was conducted between March and May 2019 in Budapest, Hungary. The three months reflect the three intervention sessions during the study, i.e. each session lasted for 4 weeks. The weather between March and May in Hungary was comfortable for both outdoor and indoor exercise.

\section{Participants}

The eligibility criteria for joining the study were adults who are between 18 and 40 years old and living in a sedentary lifestyle without reporting with clinical diseases. Two questions were asked as inclusion criteria: 1 . Do you participate in any kind of regular exercise programs (weight-lifting, walking, running, swimming etc.)? 2. Are you involved in any physical or sport teams? Either of these two questions was answered with "Yes", the volunteer was excluded from the study. The included participants were measured by Sedentary Behavior Questionnaire (SBQ), which was produced by Sedentary Behavior Research Network (SBRN). Selected participants were informed about the intervention process and agreed to wear pedometers to record their daily walking activity for four weeks. We used Omron HJ-112 (Omron Corporation, Kyoto, Japan) pedometer in the study. Participants were randomized into two groups: group A $(n=27)$ and group $\mathrm{B}(n=27)$. The random allocation was conducted according to lottery. All of the included participants were coded with number from 1 to 54, a researcher was responsible to pick one number at one time for group $\mathrm{A}$, and then for group B, consequently, the participants were grouped evenly. 


\section{Walking intervention}

The participants in Group A started 4 weeks intervention followed by a 4 weeks wash period and then 4 weeks control period, at the same timeframe, the participants in Group B started 4 weeks control followed by a 4 weeks wash period and then 4 weeks intervention period. Theoretically, the group B did not need a wash period. We decided to apply wash period to group B (1) to keep parallel with group A, (2) to avoid confounding factors such as weather, temperature, school schedule etc. The participants in the intervention group (IG) needed to carry out a 1-h aerobic walking everyday between 14:00 pm-21:00 pm. This was a robust segregation of the day. The aim was to narrow down the potential confounding factors during daytime and life rhythms (e.g., morning-type, evening-type, temperature, city intensity, daily schedule, time prior to sleep, etc.). The definition of "aerobic walking" in this intervention study were: continually walking for at least $10 \mathrm{~min}$; walk at least 60 steps per min. Participants were requested to take one hour out of their daily schedule to complete the walking task. The participants were free to choose the walking place (either on the street or in a gym), but in our study, all participants walked outdoor.

In the first session, Group A was the IG, which means the participants in group A completed daily aerobic walking; the participants in group B were asked to maintain sedentary lifestyle (i.e. control group (CG)). The second session was a wash-period. From the third session, the participants in group B started daily aerobic walking; the participants in group A continued with sedentary lifestyle. Data was collected at the beginning and end of the first and third session (see Fig. 1). Aerobic walking follows the two requirements: 1. Walk for more than 60 steps per min; 2. Continuously walking for more than $10 \mathrm{~min}$. Only when the two requirements were fulfilled together, the pedometer can record the walking data. In this study, participants who are required to carry out the intervention completed continuous 1-h daily aerobic walking on every consecutive day during 1-month period.

\section{Procedure control}

Before and after the intervention in the first and third session, questionnaire-based assessment of sleep quality was sent to the participants by email. Participants were asked to fill out the questionnaires honestly and anonymously. In line with the walking intervention, participants needed to record daily aerobic walking (DAW) associated parameters containing total steps, aerobic steps, calories, miles, starting and ending time of DAW on a diary sheet. The recorded data is for quantifying and regulating the walking process but not for statistical analysis in the present study. The variables measured in this study is elaborated below.
A researcher was responsible to collect aerobic data every day from the participants who were undergoing DAW, and the researcher also supported the DAW surveillance. All of the volunteers paused DAW in the second session, the reason of which was to enable the participants to get back to initial life status and to prepare for the third session. Thus, it increased the possibility that at the beginning of the third session, all of the participants in IG and CG were unbiased, which catered to the principle of randomization.

\section{Measurements}

We used Sedentary Behavior Questionnaire (SBQ) to assess the sedentary behaviors of the participants (not for statistical analysis). Pittsburgh Sleep Quality Index (PSQI) was used to evaluate sleep quality and sleep related components.

Nine sedentary behaviors were examined by the SBQ including television watching, playing computer/video games, sitting when listening to music, talking on the phone, doing paperwork or office work, sitting and reading, playing a musical instrument, doing arts and crafts, sitting and driving. Rosenberg, who investigated the reliability and validity of SBQ, indicated that the SBQ is an acceptable measurement for use in assessing sedentary properties [34].

Pittsburgh Sleep Quality Index (PSQI) was used to evaluate global sleep quality and seven sleep components (i.e. sleep quality, sleep latency, sleep duration, sleep disturbance, sleeping medication use, daytime dysfunction and subjective sleep) of the participants. In each sleep component, the score ranges from 0 to 3 , which follows the same scoring metric. This scale examines the sleep quality dimensions from past month, and was found with high reliability and validity [35]. The global score of PSQI ranges from 0 to 21 , the lower the score, the better the sleep quality. The borderline score of good and bad sleeper is 5, PSQI score higher than 5 indicates poor sleep quality; PSQI score lower than or equal to 5 indicates good sleep quality.

\section{Pedometer and walking dairy}

Pedometer and diary could produce better efficacy in physical activity interventions improving sleep quality [36]. Pedometers were used in this study to track the daily aerobic walking. Participants were asked to wear Omron HJ-117 for 28 consecutive days (4 weeks) in active intervention sessions. Participants who lost their pedometer during the study were provided with a new one immediately to keep the continuousness of the walking intervention. Diary was used for 


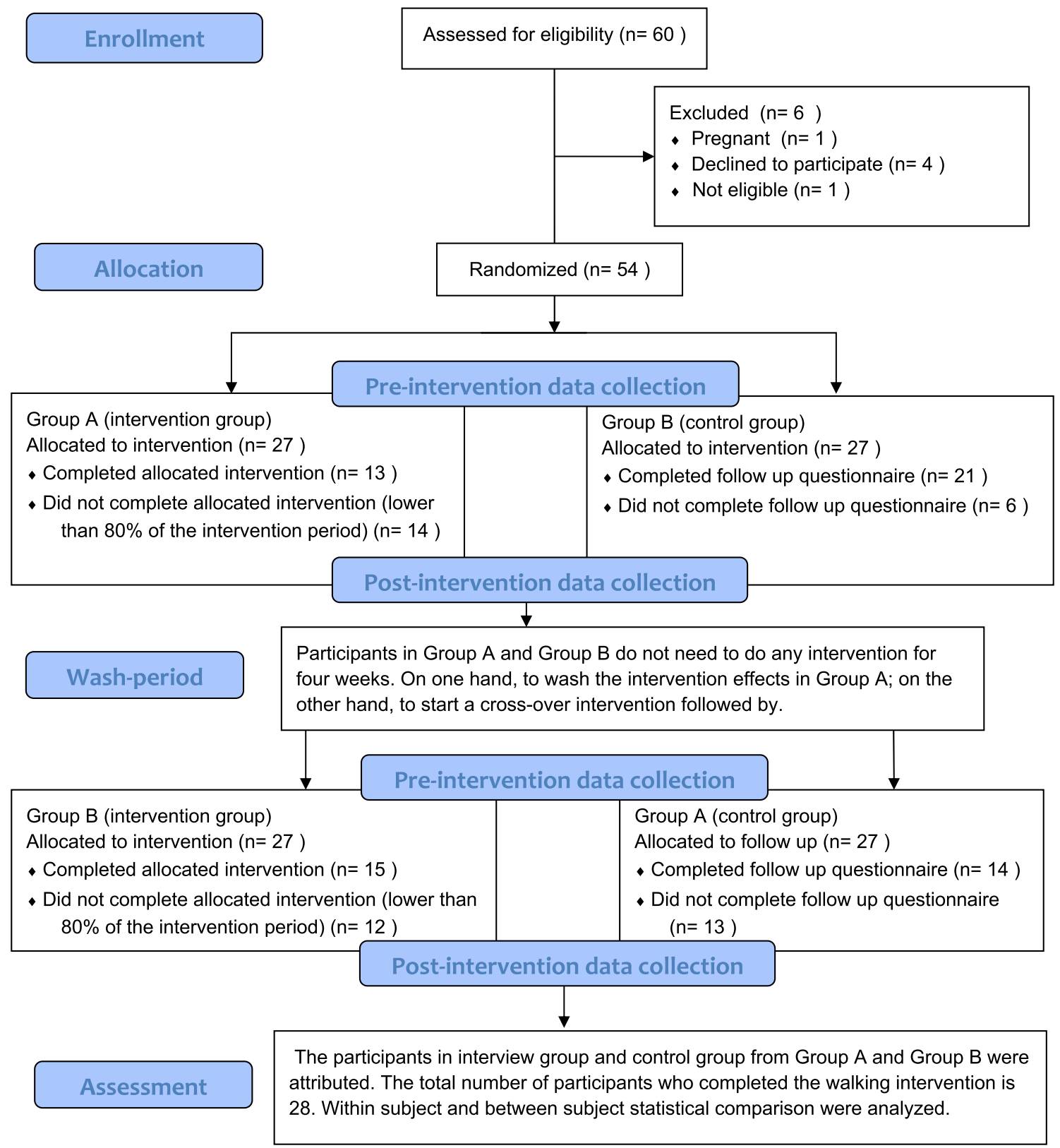

Fig. 1 The flow of the study

recording the figures from the pedometer including start \& end times, total steps, aerobic steps, calories and miles.

\section{Data analysis}

Participants who completed the walking intervention but failed to fill out the questionnaires were excluded for data analysis. To guarantee the accuracy of statistical analysis, only data from participants who fill out the questionnaire and carried out at least $80 \%$ (i.e. 22 days) of the walking days was considered for final data analysis. Independent T-test was used to compare group difference between intervention and control group. To examine the hypothesis that a DAW intervention would benefit to improve sleep quality, repeated measures ANOVA was conducted to investigate the effect of DAW pre and post intervention. The assumption of sphericity was tested using Mauchly's test, Greenhouse-Geisser and Bonferroni approach were used for correction when the model did not adequately fit. Measures of effect-size were expressed as eta-squared (ES-r) values [37]. Cohen suggested that $d=0.2$ be considered a 'small' effect size, 0.5 represents a 'medium' effect size and 0.8 a 'large' effect size [38]. The normality assumption was tested by D'AgostinoPearson Test which uses skewness and kurtosis to check 
whether the data matches normality. SPSS software (version 21.0, Chicago, USA) was used for statistical analysis; significance level was set at 0.05 .

\section{Results}

\section{Participant flow}

In total, 60 people contacted the researcher to participate the study, but 4 people were declined to participate after a detailed introduction of the intervention process. Another two people did not meet the inclusion criteria because of pregnancy and age limitation. At end, 54 participants were included in the study. We followed the CONSORT flow diagram to report the intervention process. The flow of the study process with participants is shown in Fig. 1. The total number of people who completed the intervention was 28 : Group A $(n=13)$ and Group B $(n=15)$.

\section{Demographic characteristics}

After the whole intervention period, there were 28 participants completed the walking intervention requirements as mentioned above. Age ranged from 19 to 36 years, mean age was $24.28(\mathrm{SD}=4.61)$ years. The majority of the participants were European (52\%) and Asian (40\%) university students $(n=22)$. The demographic statistics of the participants is shown in Table 1.

The skewness and kurtosis results indicated that not all research variables were perfectly normally distributed, the normally distributed variables (note: skewness and kurtosis value less than \pm 1.0 is considered normal) includes: sleep duration, sleep latency, daytime disfunction, subjective sleep, global sleep score in the first session; sleep latency, subjective sleep, global sleep score in the third session. Independent $T$-test was conducted to analyze the difference between intervention group and control group of DAW. The results in IG in the 1 st session were put together with the results in IG in the 3rd session for statistical analysis. The statistical results showed that there

Table 1 Description of the included participants

\begin{tabular}{|c|c|c|}
\hline Numerical variable & Mean \pm SD & 95\% CI (lower-upper) \\
\hline Age* & $24.28 \pm 4.61$ & $22.38-25.18$ \\
\hline Height & $171.46 \pm 7.45$ & $167.84-174.16$ \\
\hline Weight & $66.14 \pm 11.65$ & $60.33-70.22$ \\
\hline Categorical variable & $N$ & Percentage \\
\hline \multicolumn{3}{|l|}{ Gender } \\
\hline Female & 18 & $64.29 \%$ \\
\hline Male & 10 & $35.71 \%$ \\
\hline \multicolumn{3}{|l|}{ Life status* study work } \\
\hline Study & 22 & $88 \%$ \\
\hline Work & 3 & $12 \%$ \\
\hline \multicolumn{3}{|l|}{ Living condition } \\
\hline Live alone & 5 & $20 \%$ \\
\hline With relatives & 8 & $32 \%$ \\
\hline Live with flat-mates & 6 & $24 \%$ \\
\hline Live in dormitory & 6 & $24 \%$ \\
\hline \multicolumn{3}{|l|}{ Marital status* } \\
\hline Single & 21 & $84 \%$ \\
\hline Married & 4 & $16 \%$ \\
\hline \multicolumn{3}{|l|}{ Region } \\
\hline Europe & 13 & $52 \%$ \\
\hline Asia & 10 & $40 \%$ \\
\hline Middle East & 1 & $4 \%$ \\
\hline Africa & 1 & $4 \%$ \\
\hline
\end{tabular}

Categorical variables (gender, life status, living condition, marital status, region) could not be expressed by Mean $\pm \mathrm{SD}$, we inserted percentage instead in the cells

*There are missing values.

CI Confidence Interval 
was no significant difference between IG and CG regarding to sleep efficiency, sleep duration, sleep latency, sleep medication, daytime dysfunction, subjective sleep and global sleep quality $(p>0.05)$. There was a significant difference in sleep disturbance between intervention and control group $(p<0.05)$. The mean score of sleep disturbance in intervention group (Mean $=0.85, \mathrm{SD}=0.36$ ) is lower than the mean score in control group $($ Mean $=1.20$, $\mathrm{SD}=0.64$ ), which explained that sleep disturbance was significantly improved among the participants who carried out the DAW intervention. As shown in Table 2, the score of sleep efficiency (Mean $=0.30, S D=0.61$ ), sleep latency (Mean $=0.74, \mathrm{SD}=0.81)$, sleep disturbance $(\mathrm{Mean}=0.85$, $\mathrm{SD}=0.36$ ), daytime dysfunction (Mean $=0.11, \mathrm{SD}=0.58$ ), subjective sleep (Mean $=0.70, \mathrm{SD}=0.54)$ and global sleep quality (Mean $=3.59, \mathrm{SD}=2.31)$ in $\mathrm{IG}$ is lower than that in $\mathrm{CG}($ Mean $\pm \mathrm{SD}: 0.40 \pm 0.71,1.10 \pm 0.95,1.20 \pm 0.64$, $0.76 \pm 0.88,0.88 \pm 0.53,4.56 \pm 2.38$, respectively). Hence,

Table 2 Sleep components and sleep quality comparison between intervention group and control group after intervention

\begin{tabular}{|c|c|c|c|c|c|}
\hline \multirow[t]{2}{*}{ Sleep components } & \multirow{2}{*}{$\begin{array}{l}\mathrm{IG} \\
\text { Mean } \pm \mathrm{SD}\end{array}$} & \multirow{2}{*}{$\begin{array}{l}\text { CG } \\
\text { Mean } \pm \text { SD }\end{array}$} & \multicolumn{2}{|c|}{$\begin{array}{l}95 \% \text { confi- } \\
\text { dence Interval }\end{array}$} & \multirow[t]{2}{*}{$p$} \\
\hline & & & Lower & Upper & \\
\hline Sleep efficiency & $0.30 \pm 0.61$ & $0.40 \pm 0.71$ & -0.47 & 0.26 & 0.57 \\
\hline Sleep duration & $0.26 \pm 0.59$ & $0.16 \pm 0.47$ & -0.20 & 0.40 & 0.51 \\
\hline Sleep latency & $0.74 \pm 0.81$ & $1.10 \pm 0.95$ & -0.84 & 0.16 & 0.18 \\
\hline Sleep disturbance & $0.85 \pm 0.36$ & $1.20 \pm 0.64$ & -0.64 & -0.06 & $0.02 *$ \\
\hline Sleep medication & $0.11 \pm 0.58$ & $0.08 \pm 0.27$ & -0.22 & 0.28 & 0.80 \\
\hline $\begin{array}{l}\text { Daytime dysfunc- } \\
\text { tion }\end{array}$ & $0.63 \pm 0.74$ & $0.76 \pm 0.88$ & -0.58 & 0.32 & 0.57 \\
\hline Subjective sleep & $0.70 \pm 0.54$ & $0.88 \pm 0.53$ & -0.47 & 0.12 & 0.24 \\
\hline $\begin{array}{l}\text { Global sleep qual- } \\
\text { ity }\end{array}$ & $3.59 \pm 2.31$ & $4.56 \pm 2.38$ & -2.27 & 0.34 & 0.14 \\
\hline
\end{tabular}

$I G$ intervention group, $C G$ control group

Between subject comparison; *Significant $p$-value the results assumed that there was an effect of DAW on sleep and sleep components but not significant at group comparison level.

The repeated measures ANOVA with a Greenhouse-Geisser correction and Bonferroni correction were performed. The mean scores for sleep duration $(p<0.01, F$-test $=22.79)$, sleep medication $(p<0.05, F$-test $=5.22)$, subjective sleep $(p<0.05, F$-test $=5.51)$ and global sleep quality $(p<0.01$, $F$-test $=12.29)$ were statistically improved after receiving active intervention (Table 3 ). The result of daytime-disfunction $(p>0.05, F$-test $=3.15)$ was close to 0.05 , but not statistically significant. Sleep efficiency $(p>0.05, F$-test $=0.23)$, sleep latency $(p>0.05, F$-test $=0.23)$ and sleep disturbance $(p>0.05, F$-test $=1.00)$ were not statistically significantly changed. The mean score of sleep duration (Mean $=0.23$, $\mathrm{SD}=0.56)$, sleep medication $($ Mean $=0.11, \mathrm{SD}=0.49$ ), subjective sleep (Mean $=0.75, \mathrm{SD}=0.57)$ and global sleep quality (Mean $=3.89, \mathrm{SD}=2.45)$ after intervention were significantly reduced before aerobic walking intervention: sleep duration $($ Mean $=0.79, \mathrm{SD}=0.82$ ), sleep medication $($ Mean $=0.32, \mathrm{SD}=0.80)$, subjective sleep $($ Mean $=0.98$, $\mathrm{SD}=0.63)$ and global sleep quality $($ Mean $=5.20$, $\mathrm{SD}=2.96$ ). We can see that there was a significant difference in global sleep score between post-intervention and pre-intervention $(p<0.05)$. Table 3 shows the details of within subject comparison.

\section{Discussion}

This study indicates that a 4-week long DAW intervention with pedometer has beneficial effect on improving sleep and sleep components. Between group and within group comparisons suggest that the effect of DAW at group level still needs more investigation, however, the effect of DAW on individual level is significant. Better subjective sleep and overall sleep quality initiated by DAW might be considered
Table 3 Sleep components and sleep quality comparison prepost DAW intervention

\begin{tabular}{llllll}
\hline Sleep components & $\begin{array}{l}\text { Pre-intervention } \\
\text { Mean } \pm \text { SD }\end{array}$ & $\begin{array}{l}\text { Post-intervention } \\
\text { Mean } \pm \text { SD }\end{array}$ & Effect size & $F$-test & $p$ \\
\hline Sleep efficiency & $0.39 \pm 0.84$ & $0.32 \pm 0.64$ & 0.01 & 0.23 & 0.64 \\
Sleep duration & $0.79 \pm 0.82$ & $0.23 \pm 0.56$ & 0.35 & 22.79 & $0.00^{* a}$ \\
Sleep latency & $0.75 \pm 0.78$ & $0.79 \pm 0.88$ & 0.00 & 0.10 & 0.76 \\
Sleep disturbance & $1.14 \pm 0.41$ & $1.04 \pm 0.57$ & 0.02 & 1.00 & 0.32 \\
Sleep medication & $0.32 \pm 0.80$ & $0.11 \pm 0.49$ & 0.11 & 5.22 & $0.03^{*}$ \\
Daytime dysfunction & $0.84 \pm 0.83$ & $0.64 \pm 0.84$ & 0.07 & 3.15 & 0.08 \\
Subjective sleep & $0.98 \pm 0.63$ & $0.75 \pm 0.57$ & 0.14 & 5.51 & $0.02^{*}$ \\
Global sleep quality & $5.20 \pm 2.96$ & $3.89 \pm 2.45$ & 0.22 & 12.29 & $0.00^{* a}$ \\
\hline
\end{tabular}

Within subject comparison; * significant $p$-value

${ }^{\mathrm{a}} p$ value is less than 0.001 
as medicine to people who suffer from mild sleep complains to people who want to improve sleep quality.

Previous study suggested that physical activity has a therapeutic effect on improving sleep quality [39]. Regarding walking exercise to sleep quality, the study investigated the effects of outdoor walking on sleep quality, as mentioned in the introduction section that forest walking may bring improved subjective sleep quality [24]. The findings in the current study corroborated the assumption that walking might work out well in improving subjective sleep quality. The study also disclose that walking exercise may be an effective approach to sleep management which tailors to the results that the use of sleep medication decreased significantly after intervention. The reciprocal relationship between physical exercise and sleep is in line with a recent systematic review indicated that sleep and exercise exert substantial positive effects on one another [40]. The difference between indoor walking and outdoor walking was not discussed in the current study.

The group level comparison showed that sleep disturbance was decreased after walking intervention. Nowadays, sleep disturbance is highly prevalent [41]. In addition, there is a significant link between depressive symptoms and sleep disturbance [42], nevertheless, there was a remarkable relationship between sleep alterations and depression [43], aerobic walking is a recommended activity in mediating the relationship. Neuropsychological study demonstrated that physical exercise might represent a potential adjunctive treatment for neuropsychiatric disorders and cognitive impairment [44]. Furthermore, our study did not find significant effect of DAW on sleep efficiency and daytime dysfunction even though a recent study supports the notion that high exercise exertion predicts good sleep efficiency [45].

Regarding the evaluation of sleep quality, objective sleep parameters can be predicted by sleep onset latency (SOL), rapid eye movement (REM) sleep, REM latency (REM-L), stage 2, slow-wave sleep (SWS), total sleep time (TST), and wakefulness after sleep onset (WASO) [46]. The questionnaire scale (PSQI) that adopted in this study is more closely related to psychological symptom ratings [47]. The use of questionnaire to measure the sleep quality in this study was due to the reality that laboratory examination could not be applied to the big number of participants. Nevertheless, it is helpful that PSQI track sleep parameters for the past month, which was exactly coincident to the intervention period (i.e. 4 weeks). Thus, the measurement of PSQI questionnaire could possibly comply to the accuracy in measuring sleep quality compared with laboratory examination.

Exercise intensity, exercise duration and exercise time are determinant factors of sleep quality. In addition, chronotype (e.g. morning-type, evening-type), time of day may also confound the effect of exercise and sleep [48]. Additionally, studies investigate both vigorous and moderate physical exercise on sleep quality, as what has been stated in the introduction, the mainstream supports the hypothesis that evening exercise positively affects sleep, and sleep-onset latency, total sleep time, and sleep efficiency but might be impaired after vigorous exercise ending $\leq 1 \mathrm{~h}$ before bedtime [47]. However, there were also findings demonstrating that vigorous late-night exercise does not disturb sleep quality [49]. Despite the inconsistence discourse of vigorous physical activity, moderate aerobic exercise was recognized to elicit significant improvements in sleep [50].

The practical applications of the present study can be addressed from three perspectives. (1) a real-world intervention of physical exercise allows public health experts to think about not only the effectiveness of exercise but also the confounding effects caused by real-life factors when conduct exercise-based health promotion researches. (2) The current study supports the positive relationship between exercise and sleep, which shows a message for future studies to investigate the mechanism in between. (3) Exercise adherence should be concerned for physical exercise intervention studies, of which the drop off rate maybe relatively high compared with laboratory interventions. Also, factors such as weather condition and walking environment may have influential effects on exercise motivation.

Limitations exist in this study. Firstly, the number of participants who fully completed the intervention and filled out the assessment questionnaires was relatively low, which limited statistical power. Secondly, the measurement was repeatedly used during intervention process. Hence, cognitive bias may possibly appear when the participants fill out the same questionnaire in difference timepoints. Thirdly, the location of the walking intervention was not regulated, participants were free to choose to walk place. As mentioned above, walking environment may be a potential factor for walking efficiency. Last but not least, the measurement of sleep quality is self-reported, no objective data on sleep was collected.

\section{Conclusion}

Investigating feasible sleep intervention strategies for public health will produce a great impact on economic and social benefits. Daily aerobic walking elicited a significant improvement in global sleep quality from pre-intervention to post-intervention. Nevertheless, since daily aerobic walking for four weeks elicits significant improvement in sleep duration, subjective sleep and global sleep quality, and a statistically significant decrease in sleep medication use, it is highly recommended and to be implemented in public health promotion. Given the mechanism between physical exercise 
and objective sleep is uninvestigated in present study, the relationship between physiotherapy and sleep quality among young adults requires further evidence. This study could conclude that daily aerobic walking has beneficial effects on sleep quality. Further studies are suggested to examine the impact of walking intensity and walking environment on sleep quality.

Author contributions FW designed the intervention protocol, coordinated the intervention process, collected $\&$ analyzed data, and drafted the manuscript; SB proofread the manuscript, edited the language use of the manuscript and helped to finalize the manuscript. Both authors have read and approved the final version of the manuscript, and agree with the order of presentation of the authors.

Funding Open access funding provided by Eötvös Loránd University. The manuscript is without funding.

Data availability Data is available when requested with reasons.

\section{Compliance with ethical standards}

Conflict of interest The authors declare that they have no conflict of interest.

Ethics approval Approval was obtained from the ethics committee of Eötvös Loránd University. The procedures used in this study adhere to the tenets of the Declaration of Helsinki.

Informed consent Informed consent was obtained from all individual participants included in the study.

Consent for publication The participants have consented to the submission of the intervention manuscript to the journal.

Open Access This article is licensed under a Creative Commons Attribution 4.0 International License, which permits use, sharing, adaptation, distribution and reproduction in any medium or format, as long as you give appropriate credit to the original author(s) and the source, provide a link to the Creative Commons licence, and indicate if changes were made. The images or other third party material in this article are included in the article's Creative Commons licence, unless indicated otherwise in a credit line to the material. If material is not included in the article's Creative Commons licence and your intended use is not permitted by statutory regulation or exceeds the permitted use, you will need to obtain permission directly from the copyright holder. To view a copy of this licence, visit http://creativecommons.org/licenses/by/4.0/.

\section{References}

1. Young T, Peppard PE, Gottlieb DJ (2002) Epidemiology of obstructive sleep apnea: a population health perspective. Am J Respir Crit Care Med 165:1217-1239

2. Huang YS, Wang CH, Guilleminault C (2010) An epidemiologic study of sleep problems among adolescents in North Taiwan. Sleep Med 11:1035-1042
3. National Institutes of Health. What causes insomnia? https:// www.sleepfoundation.org/insomnia/what-causes-insomnia

4. Murray K, Godbole S, Natarajan L et al (2017) The relations between sleep, time of physical activity, and time outdoors among adult women. PLoS ONE 12:e0182013

5. Grandner MA (2017) Sleep, health, and society. Sleep Med Clin $12: 1-22$

6. Guimaraes LH, de Carvalho LB, Yanaguibashi G, do Prado GF (2008) Physically active elderly women sleep more and better than sedentary women. Sleep Med 9:488-493

7. Castelli L, Galasso L, Mulè A, Bruno E, Shokohyar S, Esposito F, Montaruli A, Roveda E (2020) Physical activity, chronotype and sleep in a sample of Italian elderly population. Sport Sci Health 16:55-64

8. Erlacher C, Erlacher D, Schredl M (2015) The effects of exercise on self-rated sleep among adults with chronic sleep complaints. J Sport Health Sci 4:289-298

9. King AC, Pruitt LA, Woo S, Castro CM, Ahn DK, Vitiello MV, Woodward SH, Bliwise DL (2008) Effects of moderate-intensity exercise on polysomnographic and subjective sleep quality in older adults with mild to moderate sleep complaints. J Gerontol A Biol Sci Med Sci 63:997-1004

10. Kline CE, Crowley EP, Ewing GB, Burch JB, Blair SN, Durstine JL, Davis JM, Youngstedt SD (2011) The effect of exercise training on obstructive sleep apnea and sleep quality: a randomized controlled trial. Sleep 34:1631-1640

11. Sengul YS, Ozalevli S, Oztura I, Itil O, Baklan B (2011) The effect of exercise on obstructive sleep apnea: a randomized and controlled trial. Sleep Breath 15:49-56

12. Bonardi JM, Lima LG, Campos GO et al (2016) Effect of different types of exercise on sleep quality of elderly subjects. Sleep Med 25:122-129

13. Roveda E, Vitale J, Bruno E, Montaruli A, Pasanisi P, Villarini A, Gargano G, Galasso L, Berrino F, Caumo A, Carandente F (2016) Protective effect of aerobic physical activity on sleep behavior in breast cancer survivors. Integr Cancer Sci Ther 16:21-31

14. Vitale JA, Lombardi G, Weydahl A, Banfi G (2018) Biological rhythms, chronodisruption and chrono-enhancement: the role of physical activity as synchronizer in correcting steroids circadian rhythm in metabolic dysfunctions and cancer. Chronobiol Int 35:1185-1197

15. Vuori I, Urponen H, Hasan J, Partinen M (1988) Epidemiology of exercise effects on sleep. Acta Physiol Scand 574:3-7

16. Youngstedt SD, Kripke DF (1999) Elliott JA. Is sleep disturbed by vigorous late-night exercise? Med Sci Sports Exerc 31:864-869

17. Gerber M, Brand S, Herrmann C, Colledge F, Holsboer-Trachsler E, Pühse U (2014) Increased objectively assessed vigorous-intensity exercise is associated with reduced stress, increased mental health and good objective and subjective sleep in young adults. Physiol Behav 135:17-24

18. Reynolds AC, Adams RJ (2019) Treatment of sleep disturbance in older adults. Int J Pharm Pract 49:296-304

19. Chen KM, Chen MH, Lin MH, Fan JT, Lin HS, Li CH (2010) Effects of yoga on sleep quality and depression in elders in assisted living facilities. J Nurs Res 18:53-61

20. Li F, Fisher KJ, Harmer P, Irbe D, Tearse RG, Weimer C (2004) Tai Chi and self-rated quality of sleep and daytime sleepiness in older adults: a randomized controlled trial. J Am Geriatr Soc 52:892-900

21. López-Rodríguez MM, Fernández-Martínez M, Mataran-Penarrocha GA, Rodríguez-Ferrer ME, Granados GG, Aguilar EF (2013) Effectiveness of aquatic biodance on sleep quality, anxiety and other symptoms in patients with fibromyalgia. Med Clin $141: 471-478$ 
22. Leopoldino AA, Avelar NC, Passos GB Jr et al (2013) Effect of pilates on sleep quality and quality of life of sedentary population. J Bodyw Mov Ther 17:5-10

23. Gladwell V, Kuoppa P, Tarvainen M, Rogerson M (2016) A lunchtime walk in nature enhances restoration of autonomic control during night-time sleep: results from a preliminary study. Int $\mathbf{J}$ Environ Res Public Health 13:280

24. Morita E, Imai M, Okawa M, Miyaura T, Miyazaki S (2011) A before and after comparison of the effects of forest walking on the sleep of a community-based sample of people with sleep complaints. Biopsychosoc Med 5:13

25. Tang MF, Chiu HY, Xu X et al (2019) Walking is more effective than yoga at reducing sleep disturbance in cancer patients: a systematic review and meta-analysis of randomized controlled trials. Sleep Med Rev 47:1-8

26. Suriya M, Ananda Y, Achyiar N (2017) The effectiveness of walking exercise and yoga training effect comparison to increase expiration peak toward sleep quality for COPD (Respiratory) patients in respiratory hospital West Sumatera. Adv Sci 23:12613-12615

27. Chiu HY, Huang HC, Chen PY, Hou WH, Tsai PS (2015) Walking improves sleep in individuals with cancer: a meta-analysis of randomized, controlled trials. Oncol Nurs Forum 42:E54-E62

28. Chen LJ, Fox KR, Sun WJ, Tsai PS, Ku PW, Chu D (2018) Associations between walking parameters and subsequent sleep difficulty in older adults: A 2-year follow-up study. J Sport Health Sci 7:95-101

29. Karimi S, Soroush A, Towhidi F et al (2016) Surveying the effects of an exercise program on the sleep quality of elderly males. Clin Interv Aging 11:997-1002

30. McCurry SM, Pike KC, Vitiello MV, Logsdon RG, Larson EB, Teri L (2011) Increasing walking and bright light exposure to improve sleep in community-dwelling persons with Alzheimer's Disease: results of a randomized, controlled trial. J Am Geriatr Soc 59:1393-1402

31. Grant G, Machaczek K, Pollard N, Allmark P (2017) Walking, sustainability and health: findings from a study of a walking for health group. Health Soc Care Commun 25:1218-1226

32. Christian H, Bauman A, Epping JN et al (2018) Encouraging dog walking for health promotion and disease prevention. Am J Lifestyle Med 12:233-243

33. Walsh JC, Corbett T, Hogan M, Duggan J, McNamara A (2016) An mHealth intervention using a smartphone app to increase walking behavior in young adults: a pilot study. JMIR mHealth and uHealth 4:e109

34. Rosenberg DE, Norman GJ, Wagner N, Patrick K, Calfas KJ, Sallis JF (2010) Reliability and validity of the Sedentary Behavior Questionnaire (SBQ) for adults. J Phys Act Health 7:697-705

35. Buysse DJ, Reynolds CF III, Monk TH, Berman SR, Kupfer DJ (1989) The Pittsburgh sleep quality index: a new instrument for psychiatric practice and research. Psychiatry Res 28:193-213

36. Baldursdottir B, Taehtinen RE, Sigfusdottir ID, Krettek A, Valdimarsdottir HB (2017) Impact of a physical activity intervention on adolescents' subjective sleep quality: a pilot study. Glob Health Promot 24:14-22

37. Hopkins WG, Marshall SW, Batterham AM, Hanin J (2009) Progressive statistics for studies in sports medicine and exercise science. Med Sci Sports Exerc 41:3-13

38. Cohen J (1988) Statistical power analysis for the behavioral sciences. Routledge Academic, New York

39. Reid KJ, Baron KG, Lu B, Naylor E, Wolfe L, Zee PC (2010) Aerobic exercise improves self-reported sleep and quality of life in older adults with insomnia. Sleep Med 11:934-940

40. Dolezal BA, Neufeld EV, Boland DM, Martin JL, Cooper CB (2017) Interrelationship between sleep and exercise: a systematic review. Adv Prev Med 2:1-14

41. Brown JP, Gallicchio L, Flaws JA, Tracy JK (2009) Relations among menopausal symptoms, sleep disturbance and depressive symptoms in midlife. Maturitas 62:184-189

42. Phillips KD, Moneyham L, Murdaugh C, Boyd MR, Tavakoli A, Jackson K, Vyavaharkar M (2005) Sleep disturbance and depression as barriers to adherence. Clin Nurs Res 14:273-293

43. Deslandes A, Moraes H, Ferreira C et al (2009) Exercise and mental health: many reasons to move. Neuropsychobiology 59:191-198

44. Brand S, Kalak N, Gerber M, Kirov R, Pühse U, HolsboerTrachsler E (2014) High self-perceived exercise exertion before bedtime is associated with greater objectively assessed sleep efficiency. Sleep Med 15:1031-1036

45. Youngstedt SD, Oconnor PJ, Dishman RK (1997) The effects of acute exercise on sleep: a quantitative synthesis. Sleep 20:203-214

46. Buysse DJ, Hall ML, Strollo PJ et al (2008) Relationships between the pittsburgh sleep quality index (PSQI), epworth sleepiness scale (ESS), and clinical/polysomnographic measures in a community sample. J Dent Sleep Med 4:563-571

47. Stutz J, Eiholzer R, Spengler CM (2019) Effects of evening exercise on sleep in healthy participants: a systematic review and meta-analysis. Sports Med 49:269-287

48. Vitale JA, Bonato M, La Torre A, Banfi G (2019) Heart rate variability in sport performance: do time of day and chronotype play a role? J Clin Med 8:723

49. Myllymäki T, Kyröläinen H, Savolainen K et al (2011) Effects of vigorous late-night exercise on sleep quality and cardiac autonomic activity. J Sleep Res 20:146-153

50. Passos GS, Poyares D, Santana MG, D'Aurea CV, Youngstedt SD, Tufik S, de Mello MT (2011) Effects of moderate aerobic exercise training on chronic primary insomnia. Sleep Med 12:1018-1027

Publisher's Note Springer Nature remains neutral with regard to jurisdictional claims in published maps and institutional affiliations. 